\title{
Up-regulation of long non-coding RNA SPRY4-IT1 promotes tumor cell migration and invasion in lung adenocarcinoma
}

\author{
Xia Zhang ${ }^{1}$, Qingyan $\mathrm{Chi}^{2}$ and Zhenhua Zhao ${ }^{3}$ \\ ${ }^{1}$ Department of Respiratory Internal Medicine, The People's Hospital of Linyi City, Shandong, China \\ ${ }^{2}$ Department of Neonatal, The People's Hospital of Linyi City, Shandong, China \\ ${ }^{3}$ Department of Infectious Diseases, The People's Hospital of Linyi City, Shandong, China \\ Correspondence to: Zhenhua Zhao, email: zhenhua_zhaol@yeah.net \\ Keywords: long non-coding RNA, SPRY4-ITI, lung adenocarcinoma, prognosis \\ Received: February 09, $2017 \quad$ Accepted: March 14, $2017 \quad$ Published: April 07, 2017 \\ Copyright: Zhang et al. This is an open-access article distributed under the terms of the Creative Commons Attribution License 3.0 \\ (CC BY 3.0), which permits unrestricted use, distribution, and reproduction in any medium, provided the original author and source \\ are credited
}

\section{ABSTRACT}

Long non-coding RNAs (IncRNAs) play important role in a variety of biological processes, and are tightly associated with tumorigenesis and cancer prognosis. However, the potential power of IncRNA signatures in predicting survival of patients with lung adenocarcinoma (LUAD) has not been investigated. Here, we identified a four-IncRNA signature (SPRY4-IT1, LINC00941,GPR158-AS1 and KCNK15-AS1) displaying prognostic values for LUAD using The Cancer Genome Atlas (TCGA) dataset. Based on the four-IncRNA signature, the LUAD patients can be classified into highrisk and low-risk groups with significantly different survival. We further validated the expression of SPRY4-IT1 in LUAD tissues and corresponding normal lung tissues using quantitative real-time PCR in Chinese LUAD patients. The results showed that SPRY4-IT1 was significantly up-regulated in LUAD tissues. Further analysis indicated that LUAD patients with high SPRY4-IT1 expression have significantly poorer overall survival in Chinese LUAD patients. Moreover, knock-down of SPRY4-IT1 can inhibit the lung cancer cell migration and invasion. The present work indicated that SPRY4IT1 may play a pivotal role in promoting tumor migration and invasion in LUAD. Our work implicates the promising effect of SPRY4-IT1 on the prognosis of LUAD.

\section{INTRODUCTION}

Lung cancer has become one of the most common malignancies, and the leading cause of cancer death worldwide [1]. More than 700,000 estimated new cases were diagnosed, and 60,000 patients died of lung cancer each year in China [2]. Lung adenocarcinoma (LUAD) is one of the predominant types of lung cancer in females and non-smoking males. Although therapeutic advances have significantly improved the prognosis of specific subgroups of LUAD patients, the overall survival rate of LUAD patients remains very low [3-5]. Therefore, novel cancer-specific marker is needed for LUAD patients, and it may enhance early tumor detection and treatment.

Long non-coding RNAs (lncRNA), a subclass of RNA transcripts longer than 200 nucleotides with little coding capacity, contribute to a significant regulatory information at transcriptional and epigenetic levels [69]. Accumulating evidence suggested that IncRNAs play important roles in the biological processes [10,11], and dysregulation of lncRNAs is always associated with complex diseases, including cancers [12]. Many wellknown IncRNAs have indicated tumor suppressive or oncogenic roles in various cancers, such as $H 19$ [13], HOTAIR [14], MALAT1, MEG3 [15] and PVT1 [16] etc. LncRNA expression functions as good indicator of tumor stage, implying the potential role as independent biomarkers for prognosis prediction in cancers.

Many recent works have been performed to detect lncRNA signatures to predict cancer survival [17-20], and we herein performed a comprehensive analysis for the lncRNAs expression and clinical outcome of LUAD patients based on TCGA dataset. A four-lncRNA signature displayed prognostic values for LUAD. Notably, SPRY4- 
IT1 has been reported to be desregulated in several human cancers [11, 21, 22], such as hepatocellular carcinoma [23] and melanoma [24]. However, the underlying molecular mechanism of SPRY4-IT1 remains unclear in LUAD. Here, we also examined the expression difference between LUAD tissues and normal lung tissues, and re-evaluated the clinical significance of SPRY4-IT1 in Chinese LUAD patients. Moreover, we also evaluated the effects of SPRY4-IT1 expression on tumor cell migration and invasiveness. This finding suggests the potential effects of SPRY4-IT1 expression on LUAD prognosis.

\section{RESULTS}

\section{Identification of prognostic IncRNAs associated with survival of LUAD patients}

We randomly divided 412 LUAD patients into a training dataset $(n=206)$ and a testing dataset $(n=206)$. At first, a univariate Cox regression analysis was carried out to evaluate the association between lncRNA expression and overall survival in the training dataset, and four lncRNAs were identified as prognostic lncRNAs $(P$-value $<0.001)$. Three lncRNAs (SPRY4-IT1, GPR158-AS1 and KCNK15$A S 1$ ) with higher expression were associated with shorter survival, and the remaining lncRNAs (and LINC00941) with higher expression were associated with longer survival. Next, we constructed a risk-score formula according to lncRNA expression for survival prediction as follows: Risk score $=(0.33 \times$ expression of GPR158-AS1) $+(0.12$ $\times$ expression of $K C N K 15-A S 1)+(0.27 \times$ expression value of SPRY4-IT1) + (-0.22 × expression value of LINC00941). Based on this formula, we can calculate the risk score for each LUAD patient in the training dataset, and 206 LUAD patients in the training dataset were classified into a high-

A

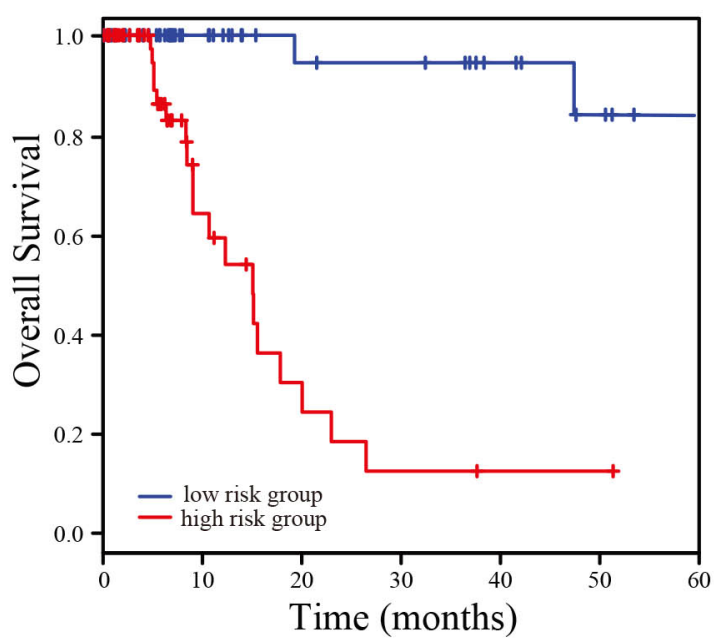

risk group $(n=103)$ and a low-risk group $(n=103)$ using the median risk score $(-1.12)$ as the threshold. The result showed a significant difference in survival between the high-risk group and the low-risk group $(P$-value $=1.3 \mathrm{E}-09$; Figure 1A). We next validated the survival prediction power of four-lncRNA signature, and 206 LUAD patients in the testing dataset were classified into a high-risk group $(\mathrm{n}=$ 126) and a low-risk group $(n=80)$ with the same threshold derived from the training dataset. The result showed that a significantly different survival between two groups $(P$-value $=2.6 \mathrm{E}-04$; Figure 1B).

Next, we evaluated whether the survival prediction power of the four-lncRNA signature was independent of other clinical variables using entire TCGA dataset. We performed multivariable Cox regression analysis, and the result showed that four-lncRNA risk score was also significantly associated with overall survival after adjusted by age, tumor stage and other clinical variables (Table 1). This result demonstrated that the survival prediction of four-lncRNA signature was independent of other clinical variables for LUAD patients.

\section{The expression of SPRY4-IT1 in LUAD tissues and cell lines}

Among these four prognostic IncRNAs, SPRY4IT1 has been widely reported in many cancer types [21, 25], whereas none of the other three lncRNAs have been reported to be associated with human cancers. So, we attempt to further examine the expression pattern of SPRY4-IT1 in LUAD patients. We measured the SPRY4IT1 expression level in the tumor tissues and adjacent normal lung tissues from 88 LUAD Chinese Han patients using quantitative RT-PCR. The result showed that SPRY4IT1 expression was significantly up-regulated in LUAD tissues (4.4 \pm 0.76$)$ compared with that in the matched

B

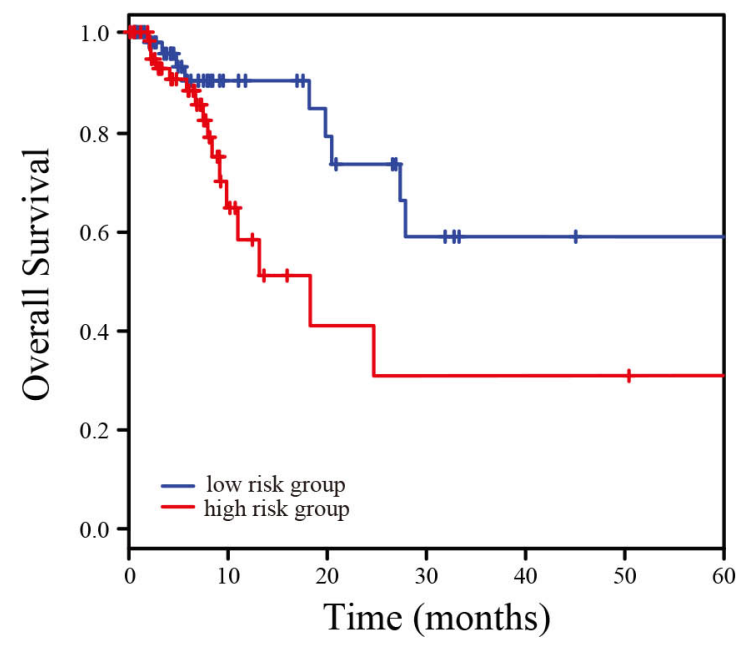

Figure 1: Kaplan-Meier estimation of overall survival of TCGA LUAD patients using the four-lncRNA signature. (A) Kaplan-Meier curves for TCGA training set; (B) Kaplan-Meier curves for TCGA testing set. 
Table 1: Multivariable Cox proportional hazard model in TCGA LUAD dataset

\begin{tabular}{lccc}
\hline \multirow{2}{*}{ Variables } & \multicolumn{3}{c}{ Multivariable analysis } \\
\cline { 2 - 4 } & HR & $\mathbf{9 5 \%}$ CI & P-value \\
\hline Age (Years) & 0.87 & $0.82-1.02$ & 0.24 \\
Gender & 0.73 & $0.47-1.49$ & 0.36 \\
Tumor size & 0.92 & $0.86-1.01$ & 0.32 \\
Tumor stage & 1.45 & $1.06-3.18$ & 0.02 \\
Risk score & 1.68 & $1.24-2.66$ & 0.001 \\
\hline
\end{tabular}

normal lung tissues $(2.9 \pm 0.88, P$-value $<0.0001$, Figure 2A). Further analysis showed that SPRY4-ITI expressions in LUAD cell lines (H23, H1299, A549 and SPC-A1) were significantly higher compared with that in the normal lung fibroblast cell line HLF (Figure 2B).

\section{Clinical significance of SPRY4-IT1 expression in LUAD}

Next, we evaluated the relationship between SPRY4IT1 expression and clinicopathological characteristics in Chinese Han LUAD patients. All these patients were divided into two groups according to the median expression level of SPRY4-IT1, including a SPRY4-ITI high-expression group $(\mathrm{n}=44)$ and a low-expression group $(\mathrm{n}=44)$. Then, the correlation between SPRY4IT1 expression and pathological factors was examined. The result indicated that patients with higherSPRY4-ITI expression had larger tumor size $(P$-value $=0.012)$ and high histological grade $(P$-value $<0.001$, Table 2$)$. Kaplan-

A)

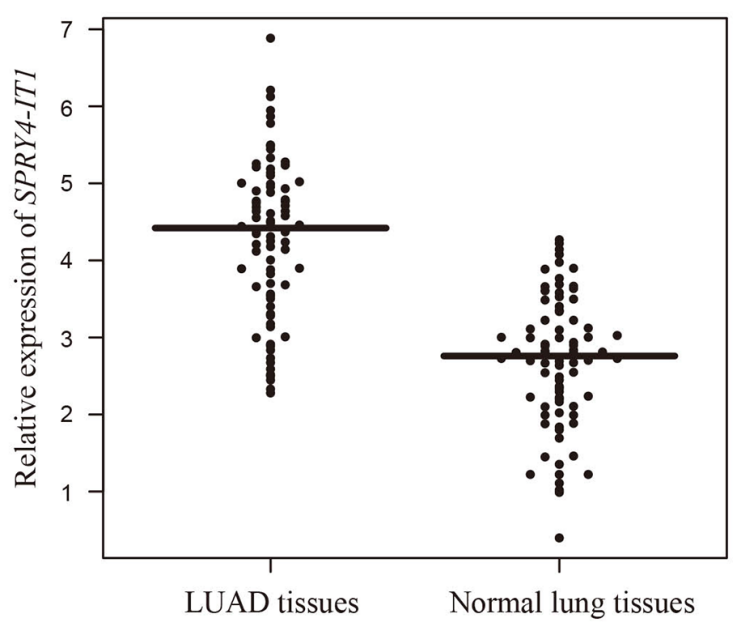

Meier survival analysis showed that the patients with higher SPRY4-IT1 expression had a significantly poorer prognosis than those with low SPRY4-IT1 expression level $(P$-value $=0.001$, Figure 3$)$. Univariate proportional hazard model showed that the histological grade and SPRY4-IT1 expression level was prognostic predictor. Multivariate analysis further indicated that histological grade $(P$-value $=0.001)$ and SPRY4-IT1 expression level $(P$-value $=0.001$, Table 3$)$ were independent prognostic factors for overall survival. This result suggests that SPRY4-IT1 might be involved in the progression of LUAD and can be used to predict overall survival in Chinese Han LUAD patients.

\section{Effect of SPRY4-IT1 on LUAD cell migration and invasion}

To investigate whether SPRY4-IT1 regulates LUAD cell migration and invasion, in vitro functional analysis were performed. The result showed that knockdown of

B)

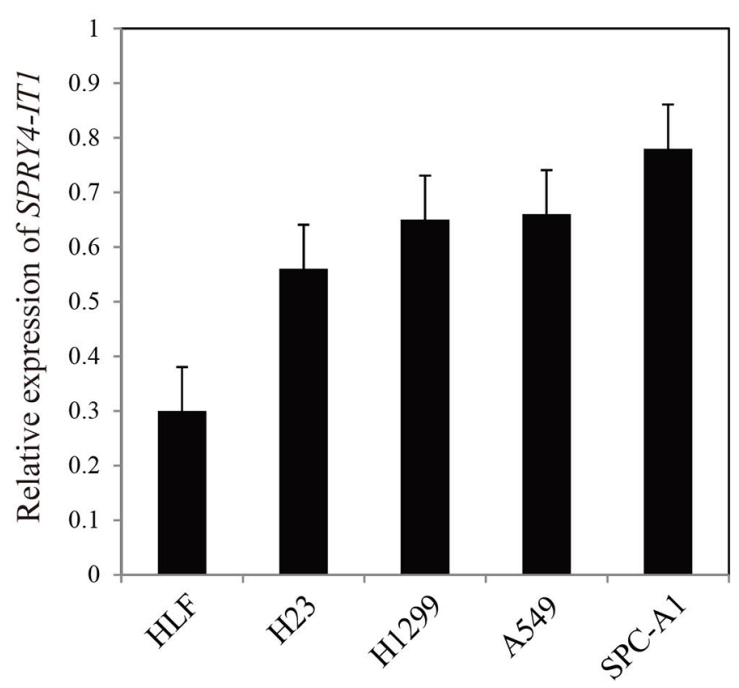

Figure 2: The relative expression levels of SPRY4-IT1 in LUAD tissues and cell lines. (A) The relative expression of SPRY4ITI in LUAD tissues and adjacent normal lung tissues. The lines represent the means of the relative expression of SPRY4-IT1. (B) The relative expression of SPRY4-IT1 in LUAD cell lines. 
Table 2: Clinicopathological associations of SPRY4-IT1 expression in Chinese Han LUAD patients

\begin{tabular}{lccc}
\hline SPRY4-IT1 expression & Low & High \\
\hline Variable & $(\mathbf{n = 4 4 )}$ & $\begin{array}{c}\text { P-value } \\
(\mathbf{n = 4 4 )}\end{array}$ & 0.88 \\
\hline Ages (years) & 21 & 18 & \\
$\quad<50$ & 23 & 26 & 0.28 \\
$\geq 50$ & & & \\
Gender & 19 & 24 & 0.002 \\
Male & 25 & 20 & \\
Female & & 13 & 0.33 \\
Tumor size & 20 & 31 & \\
$<3$ cm & 24 & & $<0.001$ \\
$\geq 3$ cm & & 15 & \\
Smoking history & 18 & 29 & \\
Non-smoker & 26 & & \\
smoker & & 7 & \\
Histological grade & 11 & 10 & \\
G1 & 15 & 27 & \\
G2 & 18 & & \\
G3 & & & \\
\hline
\end{tabular}

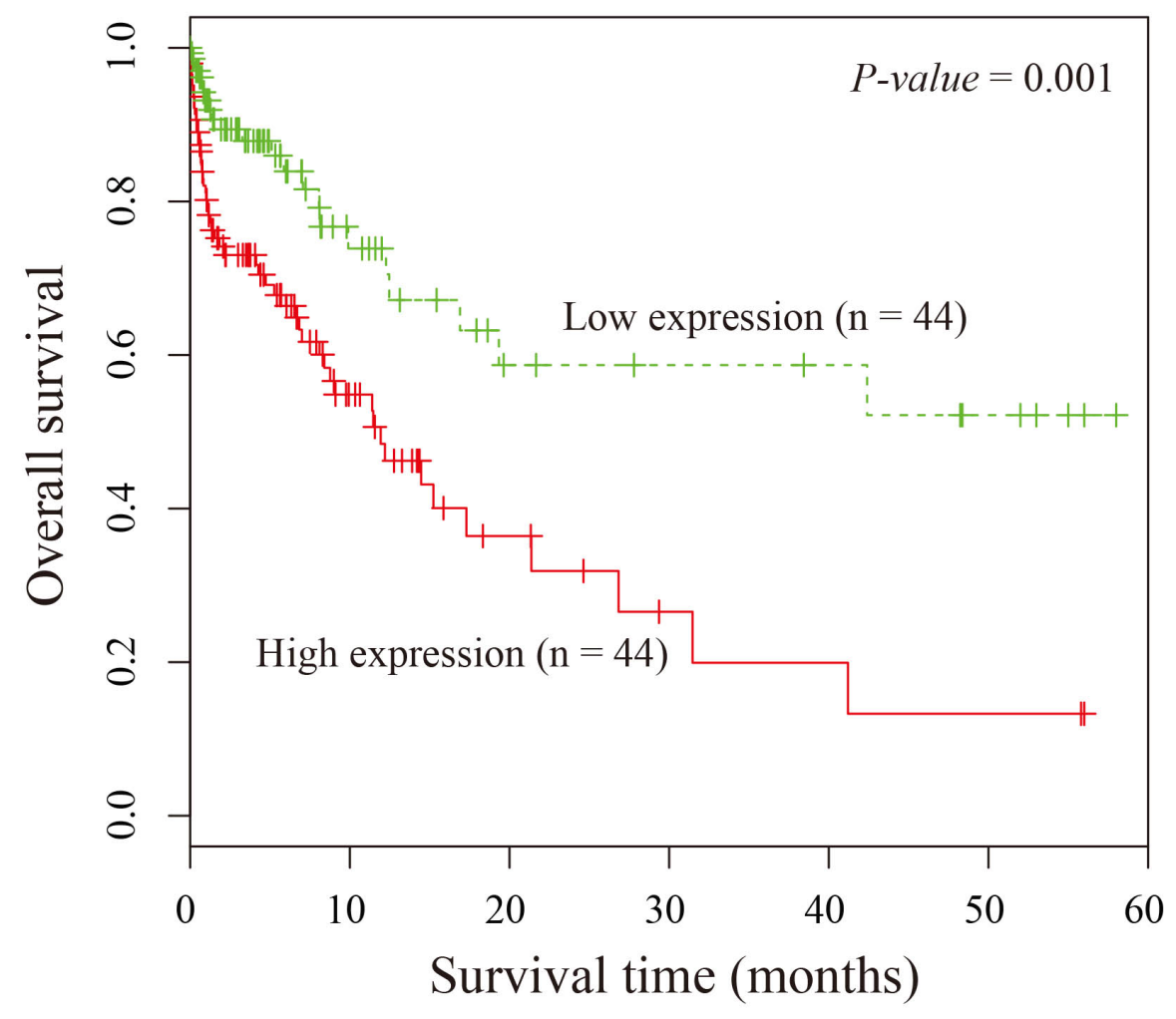

Figure 3: Kaplan-Meier survival curves of patients with LUAD based on SPRY4-IT1 expression. Patients in the higher expression group have significantly poorer prognosis than those in the lower expression group. 
Table 3: Univariate and multivariate analyses of prognostic factors in LUAD

\begin{tabular}{|c|c|c|c|c|c|c|}
\hline \multirow{2}{*}{ Variables } & \multicolumn{3}{|c|}{ Univariate analysis } & \multicolumn{3}{|c|}{ Multivariate analysis } \\
\hline & Risk & $95 \%$ CI & P-value & Risk & $95 \% \mathrm{CI}$ & P-value \\
\hline Age (Years) & 1.07 & $0.94-1.42$ & 0.55 & 1.02 & $0.92-1.28$ & 0.61 \\
\hline Gender & 1.18 & $0.83-1.68$ & 0.34 & 1.03 & $0.78-1.49$ & 0.66 \\
\hline Tumor size & 1.11 & $0.82-1.34$ & 0.09 & 1.16 & $0.96-2.48$ & 0.22 \\
\hline Histological grade & 2.66 & $1.12-4.33$ & $<0.001$ & 2.05 & $1.11-4.08$ & 0.001 \\
\hline SPRY4-IT1 & 2.21 & $1.16-3.88$ & $<0.001$ & 1.98 & $1.14-3.92$ & 0.001 \\
\hline
\end{tabular}

SPRY4-IT1 in A549 cell by RNAi significantly inhibit cell migration and invasion (Figure 4), suggesting that SPRY4IT1 gene may function as an oncogene and promote the LUAD cell migration and invasion.

\section{DISCUSSION}

LUAD is one of the most common tumors with a relative lower overall survival rate. Prognostic factor detection in LUAD patients is essential to predict patients' survival and determine optimal therapeutic strategies. Recently, many efforts have been devoted to detect specific potential markers with prognosis in LUAD [26]. As a novel biomarker, the potential roles of long non-coding RNAs in predicting prognosis have been extensively identified. Until now, nearly more than 10000 human IncRNAs have been identified, which regulate most of the genes in human genome [9]. Due to the crucial role of lncRNAs regulation at the transcription level, lncRNAs are involved in many important biological functions, such as differentiation and proliferation. Furthermore, lncRNAs show aberrant expression patterns in various cancer types, and dysregulation of lncRNAs may influence the neoplastic progression and prognosis of human malignancies [27-29].

Using TCGA LUAD dataset, we detected a fourlncRNA signature in LUAD patients to predict overall survival. Among these four prognostic lncRNAs, SPRY4-IT1 aroused our great interests. SPRY4-IT1 is nested in the intron of the SPRY4 gene. The expression

A)

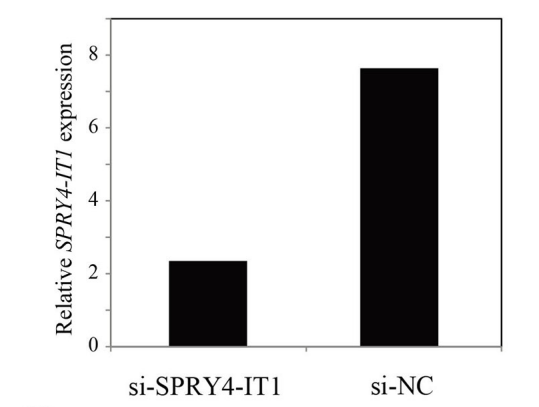

B)
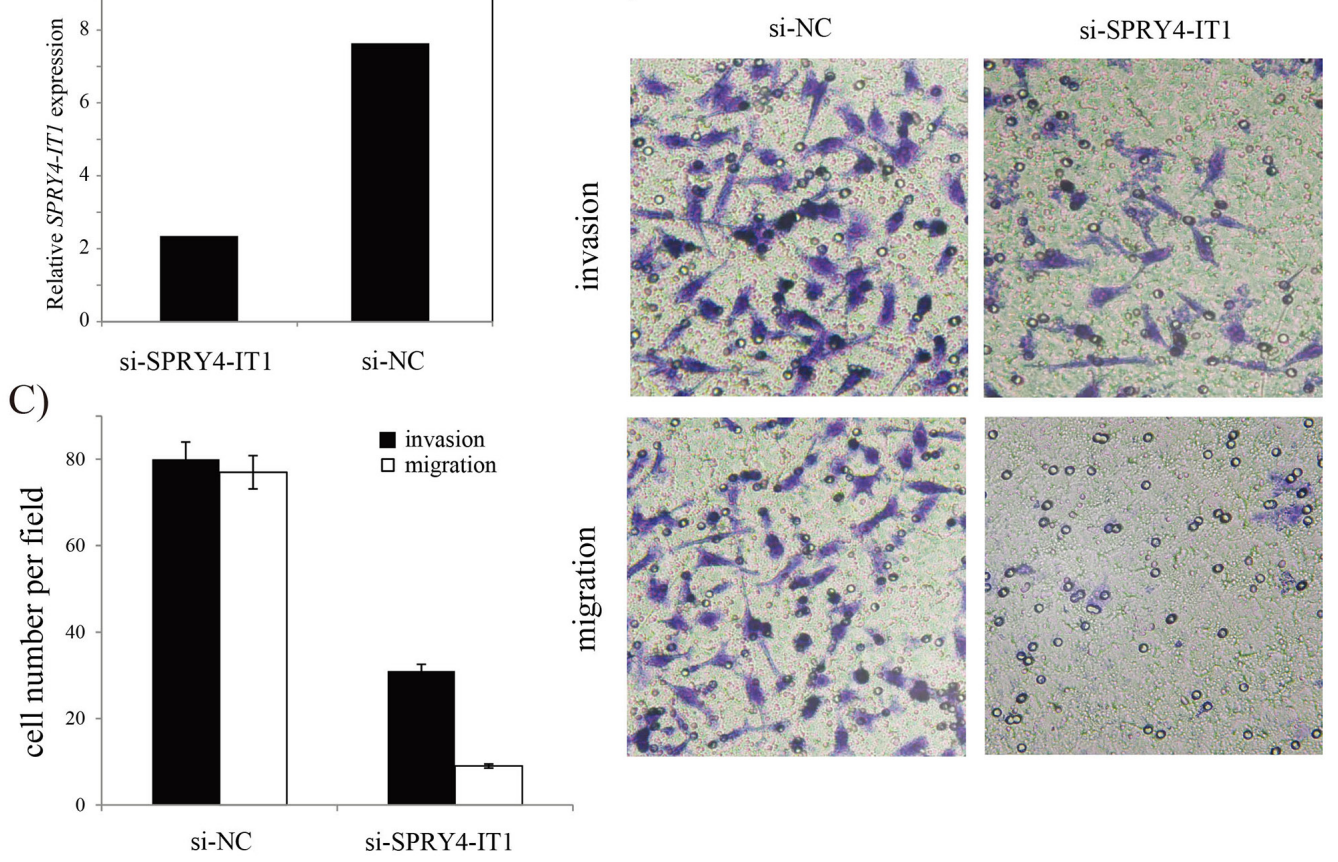

Figure 4: Effects of SPRY4-IT1 on migration and invasion of A549 cell line. (A) qRT-PCR analysis of SPRY4-IT1 in A549 cells. (B) Invasion and migration assays of A549 cells and representative fields of migration and invasive cells. (C) Average number of invasive and migration cells per field from three independent experiments. 
level of SPRY4-IT1 is highly correlated with SPRY4 gene in many cancer types, suggesting that it plays an important regulatory role during the expression of SPRY4 gene. Dysregulation of SPRY4-IT1 has been reported to perform an important function in diverse cancer types, such as hepatocellular carcinoma and melanoma, etc. [22-24, 30]. The knockdown of SPRY4IT1 expression results in defects in cell growth, decreased invasion, and increased rates of apoptosis in melanoma cells [30]. However, the underlying molecular mechanism of SPRY4-IT1 in LUAD remains unclear. In this work, we investigated the expression level of SPRY4-IT1 in LUAD and adjacent normal tissues. The results indicated that the expression of SPRY4-IT1 was extensively up-regulated in LUAD tissues and cell lines. Further analysis showed that SPRY4-IT1 could be used as a potential prognostic biomarker for LUAD. Functional characterization showed that knockdown of SPRY4-IT1 could significantly inhibit cell migration and invasion, which indicates that SPRY4-IT1 might function as a oncogene in LUAD patients.

Taken together, our present work showed that SPRY4-IT1 expression was up-regulated in LUAD and is tightly associated with cancer cell migration and invasion. Our data implicated for the first time that SPRY4-ITI expression was an independent prognostic factor of LUAD patients.

\section{MATERIALS AND METHODS}

\section{TCGA LUAD datasets and IncRNA expression}

The lncRNA expression dataset and corresponding clinical information of LUAD patients were derived from TCGA data portal (https://gdc-portal.nci.nih.gov/), and a total of 412 patients were involved in this work. The lncRNAs derived from TCGA were all annotated in GENCODE database [31] after removing the redundancy. All lncRNAs with their expression value lower than 0.3 were removed, and we finally obtained 14720 lncRNAs in 412 LUAD patients. The lncRNA expression level were defined as those with an average Reads Per Kilobase per Million mapped reads (RPKM) $\geq 0.1$ across all 412 LUAD patients. Finally, we obtained expression profiles of 12730 lncRNAs in 412 LUAD patients.

\section{Statistical analysis}

The 412 LUAD patients were randomly divided into a training set $(n=206)$ and testing set $(n=206)$. At first, the association between lncRNA expression and overall survival of LUAD patients was measured based on the training dataset using a univariate Cox regression analysis with a permutation test. Those lncRNAs with their P-values $<0.001$ were considered to be prognostic
lncRNAs. Then, a risk score model was constructed by including those prognostic lncRNAs, weighted by the estimated regression coefficients in the multivariable Cox regression analysis in the training set. Based on the risk score formula, LUAD patients can be classified into high-risk and low-risk groups for further Kaplan-Meier estimation.

\section{Patients and tissue samples}

Surgical specimens of tumor tissues and paired normal adjacent tissues were obtained from LUAD patients who underwent surgery at the people's Hospital of Linyi City (Shandong Province, China) between April 2008 and Nov 2014. Informed written consents were obtained from all enrolled LUAD patients, and all LUAD patients had a complete five-year follow-up. All patients had never received any radiotherapy before surgery excision. All tissue samples were immediately frozen in liquid nitrogen after surgery.

\section{RNA extraction and RT-PCR}

Total RNAs were extracted from tissues and cell lines using TRIzol reagent (Invitrogen, Carlsbad, USA) according to the manufacturer's protocol. The primer sequences of SPRY4-IT1 and U6 were purchased from Applied Biosystems (ABI, Foster City, CA, USA). The sequence of primers were as following: SPRY4-IT1 (forward: 5'-AGCCACATAAATTCAGCAGA-3', reverse: 5'-GATGTAGTAGGATTCCTTTCA-3'). ABI 7500 was used to performed the qPCR work.

\section{Cell lines}

Four human LUAD cell lines (H23, H1299, A549 and SPC-A1) and the human lung fibroblast cell line (HLF) were obtained from the American Type Culture Collection (Manassas, VA), and were cultured in RPMI 1640 medium (Gibco, USA) supplemented with 10\% FBS.

\section{siRNA and plasmids DNA transfections}

Small interfering RNA against SPRY4-ITI and nonspecific control siRNA were synthesized and transfected into cells using Lipfectamine 2000 transfection reagent. The sequence for the si-SPRY4IT1 was as following: si-SPRY4-IT1 (CCCAGAATGTT GACAGCTGCCTCTT).

\section{Cell migration and invasion assays}

Cell migration and invasion ability was evaluated using specialized transwell chambers $(8 \mu \mathrm{m}$ pore; $\mathrm{BD}$ Biosciences). For invasion assay, a total of $2 \times 10^{5}$ cells were added to the upper compartment of the chamber pre-coated with matrigel matrix. Medium containing 
$10 \%$ fetal bovine serum (FBS) was added to the lower chamber. After incubation for 24 hours, the non-invaded cells were removed from the upper surface with a cotton wool. The invasive cells were fixed with methanol, and then counted under a microscope. For migration assay, cells were added into upper chamber without pre-coated matrigel matrix. Images were captured by a microscope ( 5 independent fields per chamber), and the cells were counted blindly.

\section{Author contributions}

Conceived and designed the experiments: ZZ; Performed the experiments: XZ, QC, ZZ; Wrote the paper: ZZ.

\section{Ethical statements}

Patients were identified from a prospective institutional database and the study was approved by the local Research Ethics Committee.

\section{ACKNOWLEDGMENTS}

The results published here are in part based on data generated by the TCGA database: https://tcga-data.nci.nih. gov/.

\section{CONFLICTS OF INTEREST}

The authors declare no conflicts of interest.

\section{REFERENCES}

1. Torre LA, Bray F, Siegel RL, Ferlay J, Lortet-Tieulent J, Jemal A. Global cancer statistics, 2012. CA Cancer J Clin. 2015; 65: 87-108.

2. She J, Yang P, Hong Q, Bai C. Lung cancer in China: challenges and interventions. Chest. 2013; 143: 1117-1126.

3. Lin S, Sun JG, Wu JB, Long HX, Zhu CH, Xiang T, Ma H, Zhao ZQ, Yao Q, Zhang AM, Zhu B, Chen ZT. Aberrant microRNAs expression in CD133(+)/CD326(+) human lung adenocarcinoma initiating cells from A549. Mol Cells. 2012; 33: 277-283.

4. Capaccione KM, Hong X, Morgan KM, Liu W, Bishop JM, Liu L, Markert E, Deen M, Minerowicz C, Bertino JR, Allen T, Pine SR. Sox9 mediates Notch1-induced mesenchymal features in lung adenocarcinoma. Oncotarget. 2014; 5: 3636-3650. doi: 10.18632/ oncotarget. 1970.

5. Kim MH, Kim YK, Shin DH, Lee HJ, Shin N, Kim A, Lee JH, Choi KU, Kim JY, Lee CH, Sol MY. Yes associated protein is a poor prognostic factor in well-differentiated lung adenocarcinoma. Int J Clin Exp Pathol. 2015; 8: 15933-15939.
6. Kornienko AE, Guenzl PM, Barlow DP, Pauler FM. Gene regulation by the act of long non-coding RNA transcription. BMC Biol. 2013; 11: 59.

7. Cao J. The functional role of long non-coding RNAs and epigenetics. Biol Proced Online. 2014; 16: 11.

8. Li S. Expression of concern: the functional role of long noncoding RNAs and epigenetics. Biol Proced Online. 2016; 18: 12 .

9. Mercer TR, Dinger ME, Mattick JS. Long non-coding RNAs: insights into functions. Nat Rev Genet. 2009; 10: 155-159.

10. Moran VA, Perera RJ, Khalil AM. Emerging functional and mechanistic paradigms of mammalian long non-coding RNAs. Nucleic Acids Res. 2012; 40: 6391-6400.

11. Gibb EA, Brown CJ, Lam WL. The functional role of long non-coding RNA in human carcinomas. Mol Cancer. 2011; 10: 38 .

12. Cheetham SW, Gruhl F, Mattick JS, Dinger ME. Long noncoding RNAs and the genetics of cancer. Br J Cancer. 2013; 108: 2419-2425.

13. Luo M, Li Z, Wang W, Zeng Y, Liu Z, Qiu J. Long noncoding RNA H19 increases bladder cancer metastasis by associating with EZH2 and inhibiting E-cadherin expression. Cancer Lett. 2013; 333: 213-221.

14. Gupta RA, Shah N, Wang KC, Kim J, Horlings HM, Wong DJ, Tsai MC, Hung T, Argani P, Rinn JL, Wang Y, Brzoska P, Kong B, et al. Long non-coding RNA HOTAIR reprograms chromatin state to promote cancer metastasis. Nature. 2010; 464: 1071-1076.

15. Braconi C, Kogure T, Valeri N, Huang N, Nuovo G, Costinean S, Negrini M, Miotto E, Croce CM, Patel T. microRNA-29 can regulate expression of the long non-coding RNA gene MEG3 in hepatocellular cancer. Oncogene. 2011; 30: 4750-4756.

16. Colombo T, Farina L, Macino G, Paci P. PVT1: a rising star among oncogenic long noncoding RNAs. Biomed Res Int. 2015; 2015: 304208.

17. Zhang XQ, Sun S, Lam KF, Kiang KM, Pu JK, Ho AS, Lui WM, Fung CF, Wong TS, Leung GK. A long noncoding RNA signature in glioblastoma multiforme predicts survival. Neurobiol Dis. 2013; 58: 123-131.

18. Zhou M, Xu W, Yue X, Zhao H, Wang Z, Shi H, Cheng L, Sun J. Relapse-related long non-coding RNA signature to improve prognosis prediction of lung adenocarcinoma. Oncotarget. 2016; 7: 29720-29738. doi: 10.18632/ oncotarget.8825.

19. Hu Y, Chen HY, Yu CY, Xu J, Wang JL, Qian J, Zhang X, Fang JY. A long non-coding RNA signature to improve prognosis prediction of colorectal cancer. Oncotarget. 2014; 5: 2230-2242. doi: 10.18632/oncotarget.1895.

20. Bao Z, Zhang W, Dong D. A potential prognostic lncRNA signature for predicting survival in patients with bladder urothelial carcinoma. Oncotarget. 2017; 8: 10485-10497. doi: 10.18632/oncotarget.14441. 
21. Cao D, Ding Q, Yu W, Gao M, Wang Y. Long noncoding RNA SPRY4-IT1 promotes malignant development of colorectal cancer by targeting epithelial-mesenchymal transition. Onco Targets Ther. 2016; 9: 5417-5425.

22. Cui F, Wu D, He X, Wang W, Xi J, Wang M. Long noncoding RNA SPRY4-IT1 promotes esophageal squamous cell carcinoma cell proliferation, invasion, and epithelial-mesenchymal transition. Tumour Biol. 2016; 37: 10871-10876.

23. Jing W, Gao S, Zhu M, Luo P, Jing X, Chai H, Tu J. Potential diagnostic value of lncRNA SPRY4-IT1 in hepatocellular carcinoma. Oncol Rep. 2016; 36: 1085-1092.

24. Liu T, Shen SK, Xiong JG, Xu Y, Zhang HQ, Liu HJ, Lu ZG. Clinical significance of long noncoding RNA SPRY4-IT1 in melanoma patients. FEBS Open Bio. 2016; 6: 147-154.

25. Shi Y, Li J, Liu Y, Ding J, Fan Y, Tian Y, Wang L, Lian Y, Wang K, Shu Y. The long noncoding RNA SPRY4-IT1 increases the proliferation of human breast cancer cells by upregulating ZNF703 expression. Mol Cancer. 2015; 14: 51.

26. Garofalo M, Croce CM. microRNAs: master regulators as potential therapeutics in cancer. Annu Rev Pharmacol Toxicol. 2011; 51: 25-43.
27. Zhu X, Tian X, Yu C, Shen C, Yan T, Hong J, Wang Z, Fang JY, Chen H. A long non-coding RNA signature to improve prognosis prediction of gastric cancer. Mol Cancer. 2016; 15: 60 .

28. Ren YK, Xiao Y, Wan XB, Zhao YZ, Li J, Li Y, Han GS, Chen XB, Zou QY, Wang GC, Lu CM, Xu YC, Wang YC. Association of long non-coding RNA HOTTIP with progression and prognosis in colorectal cancer. Int J Clin Exp Pathol. 2015; 8: 11458-11463.

29. Ma B, Liao T, Wen D, Dong C, Zhou L, Yang S, Wang Y, Ji Q. Long intergenic non-coding RNA 271 is predictive of a poorer prognosis of papillary thyroid cancer. Sci Rep. 2016; 6: 36973.

30. Mazar J, Zhao W, Khalil AM, Lee B, Shelley J, Govindarajan SS, Yamamoto F, Ratnam M, Aftab MN, Collins S, Finck BN, Han X, Mattick JS, et al. The functional characterization of long noncoding RNA SPRY4-IT1 in human melanoma cells. Oncotarget. 2014; 5: 8959-8969. doi: 10.18632/oncotarget.1863.

31. Harrow J, Frankish A, Gonzalez JM, Tapanari E, Diekhans M, Kokocinski F, Aken BL, Barrell D, Zadissa A, Searle S, Barnes I, Bignell A, Boychenko V, et al. GENCODE. the reference human genome annotation for The ENCODE Project. Genome Res. 2012; 22: 1760-1774. 